\title{
Time-Resolved Spectroscopy of Ultrafast Photoisomerization of Octopus Rhodopsin under Photoexcitation
}

\author{
Atsushi Yabushita, ${ }^{* \dagger}{ }^{\dagger}$ Takayoshi Kobayashi, ${ }^{\dagger, \ddagger, \S, \|}$ and Motoyuki Tsuda ${ }^{\perp}$ \\ ${ }^{\dagger}$ Department of Electrophysics, National Chiao-Tung University, Hsinchu 300, Taiwan \\ ¥CREST, Japan Science and Technology Agency, 4-1-8, Honcho, Kawaguchi, Saitama 332-0012, Japan \\ ${ }^{\S}$ Department of Applied Physics and Chemistry and Institute for Laser Science, University of Electro-Communications, 1-5-1, \\ Chofugaoka, Chofu, Tokyo 182-8585, Japan \\ "Institute of Laser Engineering, Osaka University, 2-6 Yamada-oka, Suita, Osaka 565-0971, Japan \\ ${ }^{\perp}$ Kagawa School of Pharmaceutical Sciences, Tokushima Bunri University, 1314-1 Shido, Sanuki, Kagawa 769-2193, Japan
}

ABSTRACT: A primary process in vision is the cis-trans photoisomerization of a chromophore of rhodopsin, called retinal. In the present work, we have performed ultrafast time-resolved spectroscopy of octopus rhodopsin using a sub-5-fs pulse laser. In comparison with our previous study on bacteriorhodopsin, we found that octopus rhodopsin follows similar dynamics after photoexcitation but with different time constants. Spectrogram analysis showed that a $\mathrm{C}=\mathrm{N}$ stretching mode appeared directly after photoexcitation. After reaching the conical intersection region at $80 \mathrm{fs}$, the overlapping hydrogen out-of-plane and in-plane $\mathrm{C}=\mathrm{C}-\mathrm{H}$ modes emerged as distinct peaks at $\sim 200 \mathrm{fs}$, finishing a rapid relaxation along the coordinate related with these modes. The intensities of these peaks and a $\mathrm{C}=\mathrm{C}$ stretching mode were found to be modulated at a period of $\sim 500 \mathrm{fs}$, reflecting torsional motion around the $\mathrm{C}=\mathrm{C}$ double bond before thermalization with the distribution of structural variations in the all-trans structure in

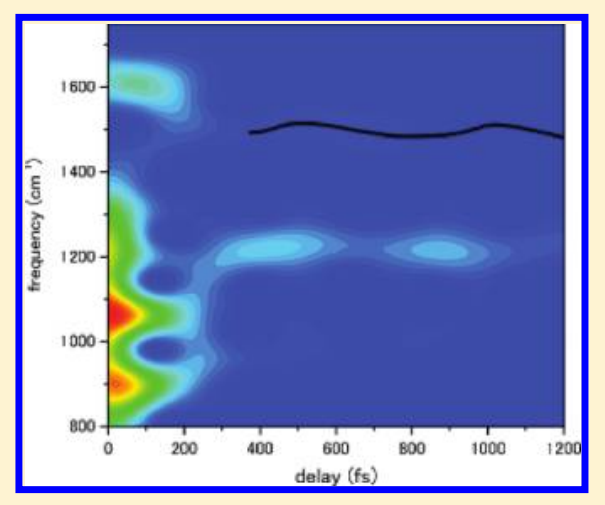
configuration space.

\section{INTRODUCTION}

The visual process in a photoactive cell consists of a series of chemical reactions mediated via several intermediates and culminating in stimulation of the optic nerve. Rhodopsin ( $\mathrm{Rh}$ ) is a photoreceptive pigment for twilight vision, which consists of the apoprotein opsin and the 11-cis-retinal chromophore. Photoexcitation of $\mathrm{Rh}$ leads to a series of intermediates that eventually initiate an enzyme cascade triggering electric excitation. ${ }^{1-5}$ The $\mathrm{Rh}$ pigment has been studied more than any other visual pigment, including those responsible for color vision, because of its relatively easy preparation. The following section describes the current understanding of the photoisomerization of retinal based on previous studies. ${ }^{6-27}$

The first intermediate of $\mathrm{Rh}$ is called prime $\mathrm{Rh}^{8}$ (or photoRh ${ }^{9}$ ) and the second intermediate, identified earlier than the first, is called bathoRh. ${ }^{2}$ Comparison of the absorption spectrum of prime $\mathrm{Rh}^{10}$ and studies of 11 -cis-locked $\mathrm{Rh}^{11,12}$ indicated that the chromophore in primeRh has a distorted alltrans configuration. Therefore, to form bathoRh, the chromophore must undergo cis-trans isomerization. Studies on the cis-trans isomerization yield of the $\mathrm{Rh}$ chromophore in solution ${ }^{13,14}$ and in protein ${ }^{15}$ have shown that the isomerization yield is enhanced by more than an order of magnitude in the protein environment. Other studies have inferred that the first intermediate (primeRh) is the nonthermal state of bathoRh. ${ }^{16,17}$ The transitions between the intermediates and their time constants have been reported as follows. After photoexcitation of $\mathrm{Rh}$, a Franck-Condon (FC) state proceeds to a conical intersection (CI) on the potential energy surface of the electronic excited state in $\sim 100$ fs as estimated in time-resolved measurements. ${ }^{6,17-20}$ Femtosecond transient absorption measurement has elucidated that curve crossing to the ground state takes about 200 fs to form the highly distorted primeRh. ${ }^{21}$ Picosecond Raman study clarified that a temporal decay of a few picoseconds corresponds to conversion from primeRh to bathoRh. $^{22}$

Relatively few studies ${ }^{18-21}$ exist on the ultrafast dynamics of $\mathrm{Rh}$ because of the difficulties in preparation due to the fragility of the samples. In place of detailed studies on $\mathrm{Rh}$, bacteriorhodopsin (bR) has been extensively studied as a model system as it is relatively robust in experiments. In the femtosecond time-resovled stimulated Raman study of $b R^{23}$ the photoisomerization of $\mathrm{bR}$ has been reported to follow the process

$$
\mathrm{bR} \stackrel{h \nu}{\rightarrow} \mathrm{FC}(\mathrm{H}) \stackrel{<200 \mathrm{fs}}{\longrightarrow} \mathrm{CI}\left(\mathrm{I}_{460}\right) \stackrel{500 \mathrm{fs}}{\longrightarrow} \mathrm{J} \stackrel{3 \mathrm{ps}}{\longrightarrow} \mathrm{K} \rightarrow \cdots
$$

In a previous work we performed ultrafast time-resolved spectroscopy of bR to clarify its electronic dynamics and

Received: September 28, 2011

Revised: December 17, 2011

Published: January 17, 2012 


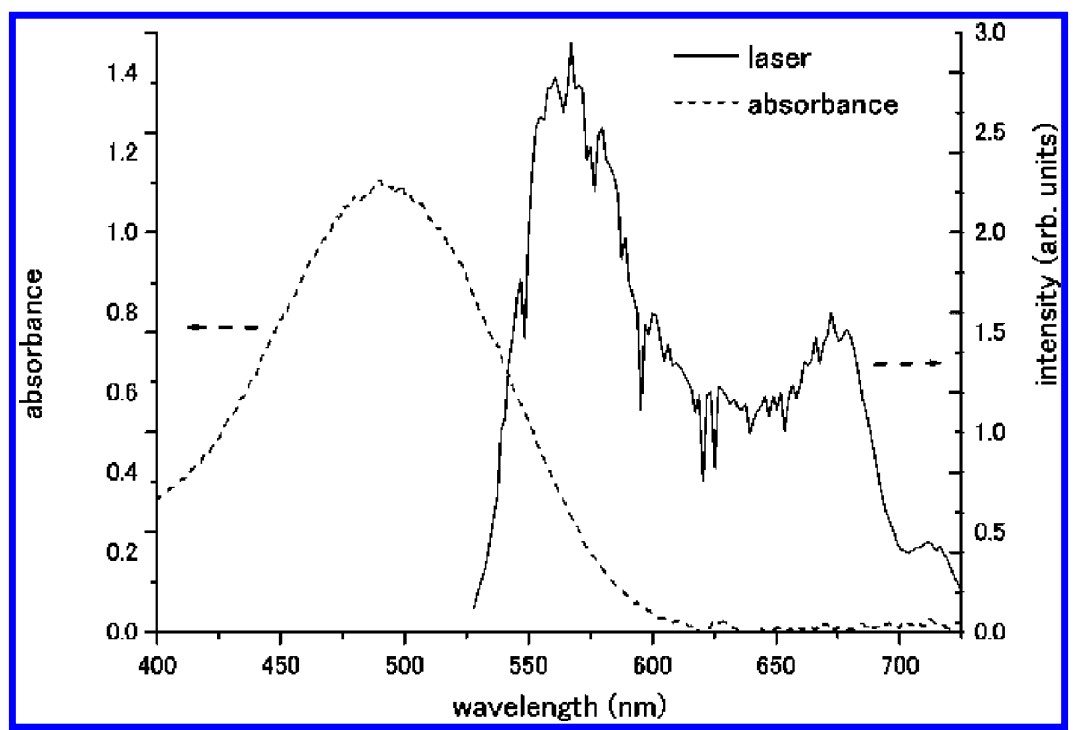

Figure 1. Laser spectrum (solid line) and absorption spectrum (dotted line) of octopus Rh.

vibration dynamics in detail. ${ }^{24}$ The results elucidated the dynamics of the intermediates, which have lifetimes consistent with previous reports on dynamic hole-burning, ${ }^{25}$ time-resolved fluorescence, ${ }^{26}$ and transient absorption. ${ }^{27}$ However, the relaxation dynamics of $\mathrm{bR}$ and $\mathrm{Rh}$ are expected to be quite different considering the differences in structural transition between the retinal in bR (from all-trans to 13-cis configuration) and that in $\mathrm{Rh}$ (from 11-cis to all-trans configuration).

In the present work, we have performed ultrafast timeresolved spectroscopy of octopus $\mathrm{Rh}$ using a sub-5-fs laser pulse and a 128-channel detection system. The ultrafast time resolution of sub-5-fs enabled us to study both the electronic and vibration dynamics, and the multichannel detector array allowed simultaneous observation of the signals over all probe wavelengths, avoiding sample degradation. The observed ultrafast dynamics of $\mathrm{Rh}$ showed a difference from the results for bR obtained in the previous work, as the present work clarified that the transition from the FC state to $\mathrm{CI}$ proceeds faster in octopus $\mathrm{Rh}$ than in $\mathrm{bR}$.

\section{EXPERIMENTAL METHODS}

Femtosecond Spectroscopy Apparatus. The pulsedlight sources and setup for femtosecond time-resolved absorption spectroscopy used in this work are described in previous reports from our group. ${ }^{28-30}$ A $4.7-\mathrm{fs}, 1-\mathrm{kHz}$ pulse train was generated from a noncollinear optical parametric amplifier (NOPA) with a pulse compressor, ${ }^{28}$ covering a wavelength range from 528 to $727 \mathrm{~nm}$. The laser spectrum obtained by NOPA is shown in Figure 1. The energy and peak intensity of the pump pulses at the sample were $\sim 10 \mathrm{~nJ}$ and $10 \mathrm{GW} / \mathrm{cm}^{2}$, respectively, which are approximately 10 times higher than the probe pulses. The pulse energies (intensities) of the pump and probe pulses at the sample position were approximately 20 and $5 \mathrm{~nJ}\left(7.5 \times 10^{14}\right.$ and $8.5 \times 10^{13}$ photons $\left./ \mathrm{cm}^{2}\right)$, respectively.

In order to measure weak pump-probe signals at various wavelengths, we used a multichannel lock-in amplifier, which was specifically designed for the simultaneous detection of lowintensity signals over the entire spectral region. The multichannel lock-in amplifier consists of 128 lock-in amplifiers connected to 128 avalanche photodiodes. In the present experiment, multichannel signals, spectrally resolved by a polychromator (JASCO M25-TP), were detected by the avalanche photodiodes with the lock-in amplifiers in reference to pump pulses modulated at $500 \mathrm{~Hz}$ by a mechanical chopper. The normalized transmittance changes in the range extending from 528 to $727 \mathrm{~nm}$ were measured for -100 to $2000 \mathrm{fs}$ with a 1 -fs interval.

Octopus Rh. The microvillar membranes of the octopus retina (Mizudako, Paroctopus dofleini) were isolated by sucrose flotation (34 wt \%, buffer A) (400 mM KLC, $10 \mathrm{mM} \mathrm{MgCl}_{2}+$ $20 \mu \mathrm{M}$ p-APMSF) repeated twice. The obtained pellet was washed several times with buffer A and with buffer B (10 mM MOPS [pH 7.4], $1 \mathrm{mM}$ DTT, $20 \mu \mathrm{M}$ p-APMSF). The final products were suspended in buffer $\mathrm{B}$ and kept at $-80{ }^{\circ} \mathrm{C}$ in the dark. A part of this frozen sample was thawed and centrifuged. The as-obtained pellet was solubilized in $\mathrm{H}_{2} \mathrm{O}$ with $2 \mathrm{mM}$ MOPS [pH 7.4] and 2\% sucrose monolaurate (L-1690 or SM1200). The solution was centrifuged, and the supernatant was used as the sample, which was added to a $1 \mathrm{~mm}$-thick stationary optical cell for experiments without circulation. The absorption spectrum of the sample showed no difference between before and after the pump-probe measurement within the margin of the error of about $10 \%$. The peak absorbance at $490 \mathrm{~nm}$ of the sample solution was 1.1. The absorption spectrum of the octopus $\mathrm{Rh}$ is plotted in Figure 1 together with the laser spectrum.

\section{RESULTS AND DISCUSSION}

Figure $2 \mathrm{a}$ shows the real-time absorption difference spectra, $\Delta A$, in the time region between -100 and 2000 fs over the spectral region from 528 to $727 \mathrm{~nm}$. Real-time traces probed at 577, 610, 641, and $682 \mathrm{~nm}$ are shown in Figure 2b. In all the observed spectral regions, the sign of $\Delta A$ was positive in the initial period following photoexcitation, which is thought to reflect induced absorption. Relaxation from the FC state (named the $\mathrm{H}$ state in the case of bR) in the first electronic excited state to a geometrically relaxed state such as a twisted state, which is called the I state in the case of bR, is associated with the decrease in the positive $\Delta A$ signal. This is induced by 


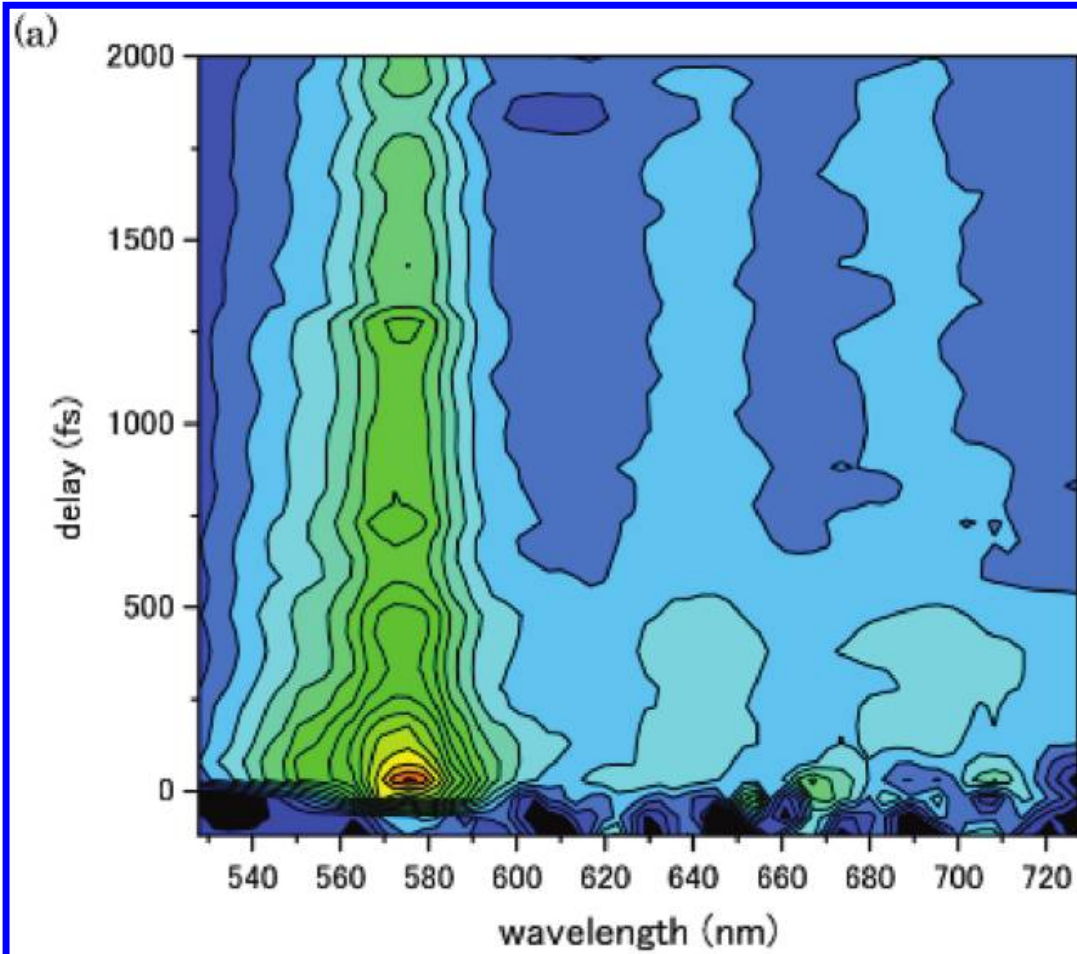

(b)
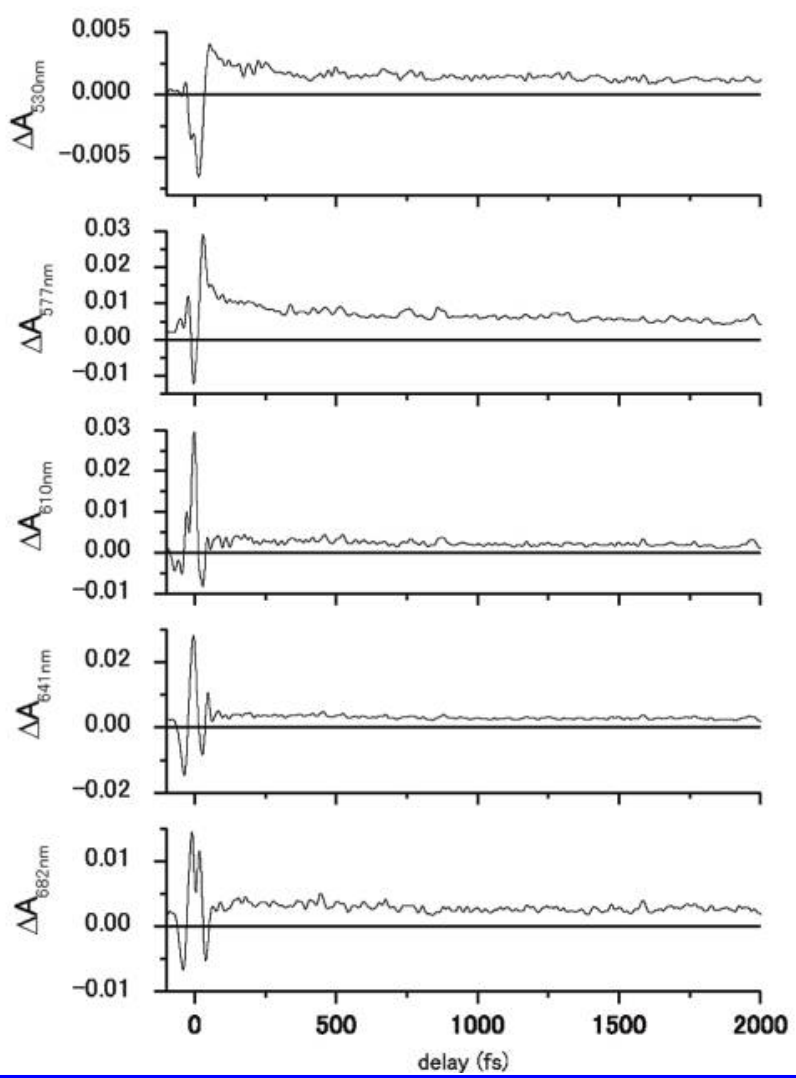

Figure 2. Observed time-resolved difference absorption spectra. (a) Two-dimensional display of measured time-resolved difference absorption spectra $\Delta A(t, \lambda)$. (b) Real-time traces of the absorbance difference for five probe wavelengths.

the spectral blue shit of the induced absorption. The shift takes place because the induced absorption is affected by the energy stabilization and destabilization of the initial state (I state in the case of $b R$ ) and the final state (FC state of the induced absorption from the I state), respectively. The oscillatory features around time zero are considered to be coherent artifacts generated by the interference between probe light and scattered pump light. 
The photoexcited $\mathrm{Rh}$ is reported to decay by the following process: $6,7,20$

$$
\mathrm{Rh} \stackrel{h \nu}{\rightarrow} \mathrm{FC} \stackrel{<100 \mathrm{fs}}{\longrightarrow} \mathrm{CI} \stackrel{100-300 \mathrm{fs}}{\longrightarrow} \text { primeRh } \stackrel{1-4 \mathrm{ps}}{\longrightarrow} \text { bathoRh } \stackrel{>1 \mathrm{~ns}}{\longrightarrow}
$$

where FC indicates the Franck-Condon state. The FC and CI were previously called the $\mathrm{H}$ state and $\mathrm{J}$ state, respectively, as described above.

Electronic Dynamics. To determine the lifetimes and spectra of the decaying components, we fitted the observed time-resolved difference absorption spectra $\Delta A(t, \lambda)$ using a global fitting method ${ }^{31}$ over all probe wavelengths. The fitting function included three components as follows, representing two transitions with distinct time constants:

$$
\begin{aligned}
\Delta A(t, \lambda)= & \Delta A_{0}(\lambda)+\Delta A_{1}(\lambda) \exp \left(-t / \tau_{1}\right) \\
& +\Delta A_{2}(\lambda) \exp \left(-t / \tau_{2}\right) \\
& \left(\tau_{1}<\tau_{2}\right)
\end{aligned}
$$

Finding the condition for least-squares error in the global fit analysis, we have estimated the time constants of $\tau_{1}$ and $\tau_{2}$ as $80 \mathrm{fs}$ and $1.1 \mathrm{ps}$, respectively. The two time constants reflect the sequential relaxation after photoexcitation. The whole process can be expressed by

$$
\begin{aligned}
\Delta A(t, \lambda)= & \Delta A_{\mathrm{a}}(\lambda) \exp \left(-t / \tau_{1}\right)+\Delta A_{\mathrm{b}}(\lambda) \\
& \times\left[1-\exp \left(-t / \tau_{1}\right)\right] \exp \left(-t / \tau_{2}\right) \\
& +\Delta A_{\mathrm{c}}(\lambda)\left[1-\exp \left(-t / \tau_{1}\right)\right] \\
& \times\left[1-\exp \left(-t / \tau_{2}\right)\right]
\end{aligned}
$$

where $\Delta A_{i}(\lambda)(i=\mathrm{a}, \mathrm{b}, \mathrm{c})$ is the spectrum of each intermediate. Considering $\tau_{1} \ll \tau_{2}$, eq 3 can be approximated as

$$
\begin{aligned}
\Delta A(t, \lambda)= & \Delta A_{\mathrm{a}}(\lambda) \exp \left(-t / \tau_{1}\right)+\Delta A_{\mathrm{b}}(\lambda) \\
& \times\left[\exp \left(-t / \tau_{2}\right)-\exp \left(-t / \tau_{1}\right)\right] \\
& +\Delta A_{\mathrm{c}}(\lambda)\left[1-\exp \left(-\mathrm{t} / \tau_{2}\right)\right]
\end{aligned}
$$

Using eqs 2 and 4, we obtain

$$
\begin{aligned}
& \Delta A_{1}(\lambda)=\Delta A_{\mathrm{a}}(\lambda)-\Delta A_{\mathrm{b}}(\lambda) \\
& \Delta A_{2}(\lambda)=\Delta A_{\mathrm{b}}(\lambda)-\Delta A_{\mathrm{c}}(\lambda) \\
& \Delta A_{0}(\lambda)=\Delta A_{\mathrm{c}}(\lambda)
\end{aligned}
$$

Equations 5-7 give

$$
\begin{aligned}
& \Delta A_{\mathrm{a}}(\lambda)=\Delta A_{0}(\lambda)+\Delta A_{1}(\lambda)+\Delta A_{2}(\lambda) \\
& \Delta A_{\mathrm{b}}(\lambda)=\Delta A_{0}(\lambda)+\Delta A_{2}(\lambda)
\end{aligned}
$$

Spectra of $\Delta A_{0}(\lambda), \Delta A_{1}(\lambda)$, and $\Delta A_{2}(\lambda)$ were estimated by least-squares fit of $\Delta A(t, \lambda)$ using the two obtained time constants of $\tau_{1}$ and $\tau_{2}$. Using eqs 7-9, $\Delta A_{\mathrm{a}}(\lambda), \Delta A_{\mathrm{b}}(\lambda)$, and $\Delta A_{c}(\lambda)$ were calculated from the spectra of $\Delta A_{0}(\lambda), \Delta A_{1}(\lambda)$, and $\Delta A_{2}(\lambda)$, and the result is plotted in Figure 3a. The three spectra were normalized for their comparison in Figure $3 \mathrm{~b}$.

Previous work by Shank and Mathies and others observed the signal rise on a $100 \mathrm{fs}$ time scale in their study of femtosecond transient absorption measured by 35 -fs pump pulses, and concluded that the wavepacket rapidly leaves the FC region in the $100 \mathrm{fs}$ time scale. ${ }^{32}$ Mathies claimed to have found that the system is carried toward CI in $\sim 50 \mathrm{fs}$ in his

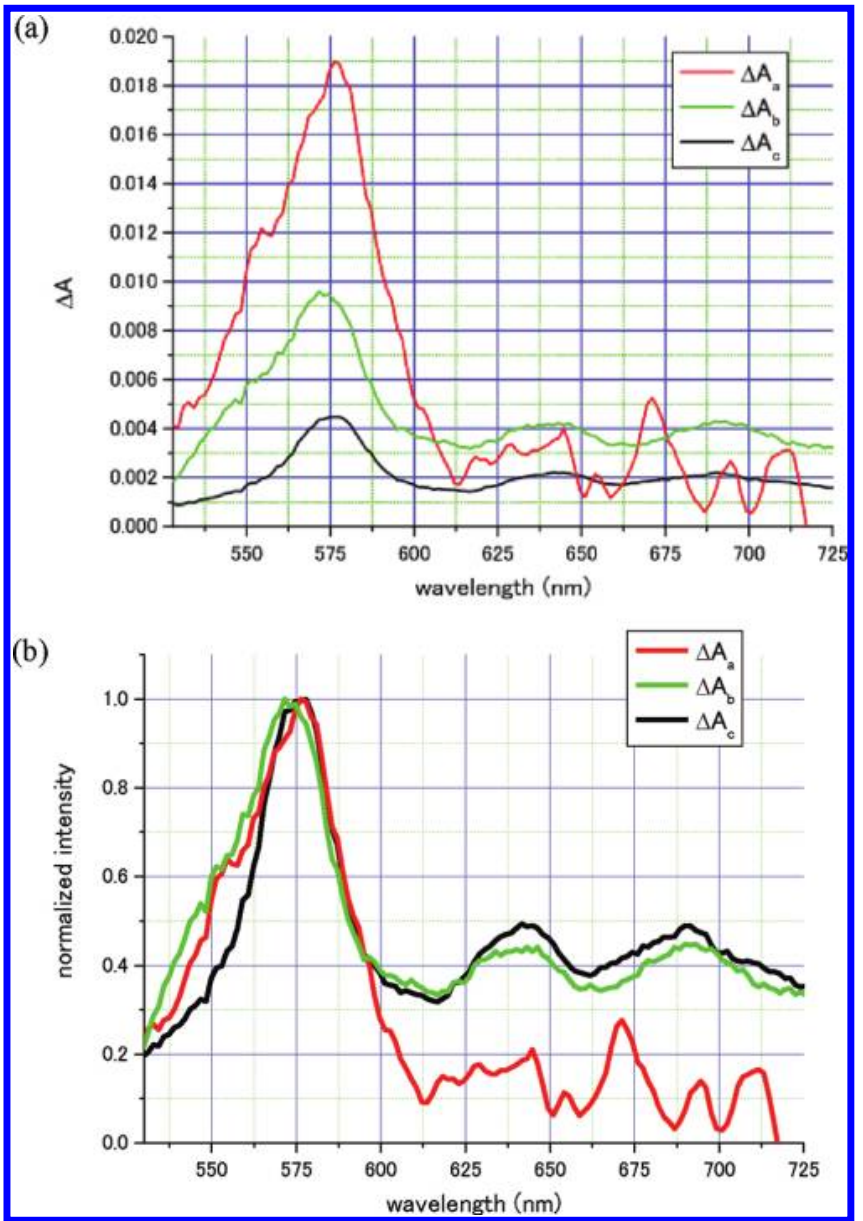

Figure 3. Results of global fitting analysis. (a) Mean square error calculated scanning the value of $\tau_{1}$ and $\tau_{2}$. (b) Spectra of $\Delta A_{\mathrm{a}}(\lambda)$, $\Delta A_{\mathrm{b}}(\lambda)$, and $\Delta A_{\mathrm{c}}(\lambda)$ calculated from the spectra of $\Delta A_{i}(\lambda)(i=0,1,2)$. The spectra of $\Delta A_{i}(\lambda)$ were obtained in least-squares fit of $\Delta A(t, \lambda)$ using $\tau_{1}$ and $\tau_{2}$ estimated in panel a.

study of femtosecond stimulated Raman spectroscopy using 30-fs photochemical pump pulses. ${ }^{7}$ It implies that $\tau_{1}$ (80 fs) obtained in the present work corresponds to the time moving from the FC region to CI. In the present work, we could determine the time constant using a much shorter pulse of sub5 -fs pulses. The spectrum of $\Delta A_{a}(\lambda)$ in eq 3 has a negative value, which is thought to reflect the induced absorption of the FC state.

Spectra of $\Delta A_{\mathrm{b}}(\lambda)$ and $\Delta A_{\mathrm{c}}(\lambda)$ show similar spectral shape with positive value in the all-probe wavelength region, reflecting induced absorption of reaction intermediates. The spectrum of $\Delta A_{\mathrm{c}}(\lambda)$ around $575 \mathrm{~nm}$ is narrower than the spectrum of $\Delta A_{\mathrm{b}}(\lambda)$. The narrowing of the spectrum is considered to be caused by the vibration cooling on the ground state potential surface. Therefore $\Delta A_{\mathrm{b}}(\lambda)$ and $\Delta A_{\mathrm{c}}(\lambda)$ are thought to correspond to the induced absorption of primeRh and bathoRh, respectively. This implies that primeRh becomes thermalized into bathoRh in the time constant of $\tau_{2}$.

Vibration Dynamics. Wavepacket motion in the electronic excited state and that in the electronic ground state modulate the $\Delta A(t)$ traces. Therefore, Fourier analysis of the $\Delta A(t)$ traces reflects the vibrational modes either of the photoactive state or the nonphotoactive state. The vibration mode signals of the nonphotoactive state are expected to decay without 

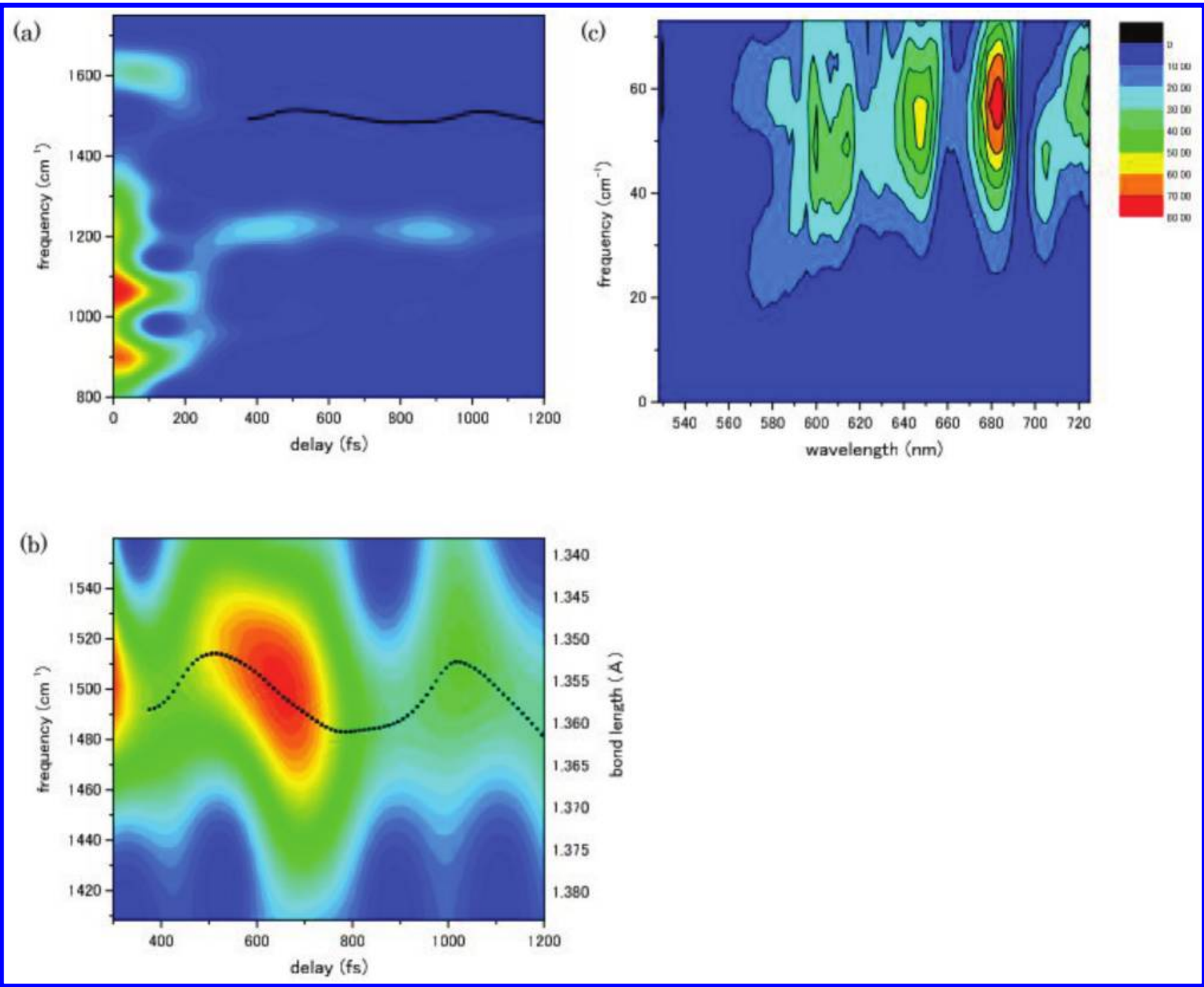

Figure 4. Spectrograms calculated from the $\Delta A$ traces at $530 \mathrm{~nm}$. The black dots show the $\mathrm{C}=\mathrm{C}$ stretching mode. Spectrograms are shown for the (a) $900-1750 \mathrm{~cm}^{-1}$ range and (b) the $1410-1560 \mathrm{~cm}^{-1}$ range rescaled in intensity to show the frequency modulation of the $\mathrm{C}=\mathrm{C}$ stretching mode. (c) Two-dimensional Fourier power spectra of the time-resolved difference absorption traces $\Delta A(t)$ over delay times ranging from 800 to 2000 fs.

frequency shift or modulation. Therefore, the vibration mode signals showing frequency shift or modulation are thought to be assigned to the dynacmics of the vibration mode coupled to the photoactive state. In the following, we discuss the vibration mode signals showing frequency shift or modulation to discuss the dynamics of the vibration mode coupled to the photoactive state

Fourier power spectra of the time traces of the absorption difference exhibit three prominent modes at around 1550, 1200 , and $1000 \mathrm{~cm}^{-1}$ assigned to $\mathrm{C}=\mathrm{C}$ stretching $\left(\nu_{\mathrm{C}=\mathrm{C}}\right)$, $\mathrm{C}-\mathrm{C}$ stretching $\left(\nu_{\mathrm{C}-\mathrm{C}}\right)$, and hydrogen out-of-plane (HOOP) modes, respectively. The dynamics of those vibration modes at each probe wavelength can be studied by calculating the instantaneous molecular vibration frequencies using spectrogram analysis ${ }^{33,34}$ of the time-resolved difference absorption traces. A Blackman window with a fwhm of $240 \mathrm{fs}$ was used as a gate function in the spectrogram calculation. Figure 4a shows the calculated spectrograms at a probe wavelength of $530 \mathrm{~nm}$. For delay times shorter than $150 \mathrm{fs}$, the spectrogram shows the existence of a $\mathrm{C}=\mathrm{N}$ stretching mode at a frequency of $\sim 1610 \mathrm{~cm}^{-1}$, which quickly decays in a similar fashion to that observed in photoisomerization of $\mathrm{bR}^{24}$ This confirms that the primary event after photoexcitation of the octopus $\mathrm{Rh}$ is a deformation of the retinal configuration near the $\mathrm{C}=\mathrm{N}$ bond of the protonated Schiff base.

Just after the photoexcitation, strain in retinal is localized around the Schiff base and propagates to surrounding $\mathrm{C}=\mathrm{C}$ bonds within a few hundred femtoseconds. Therefore the $\mathrm{C}=\mathrm{N}$ stretching mode is more distinct than the $\mathrm{C}=\mathrm{C}$ stretching mode in the early time scale. The reason why the observed frequency of the $\mathrm{C}=\mathrm{N}$ stretching mode $\left(1610 \mathrm{~cm}^{-1}\right)$ was lower than that of the ground state reported as $\sim 1650 \mathrm{~cm}^{-1}$ in Raman study $^{35}$ can be explained as follows in the analogy of the explanation for $\mathrm{bR}^{36}$ The Schiff base forms a hydrogen bond to its salt bridge partner in the ground state. The primary process after photoexcitation involves a movement of the Schiff base proton away from a counterion, which breaks the hydrogen bond and causes a red-shift of the $\mathrm{C}=\mathrm{N}$ stretching frequency.

Figure $4 \mathrm{~b}$ shows a spectrogram of the $1410-1560 \mathrm{~cm}^{-1}$ range with rescaled intensity to clearly show the frequency modulation in the $\mathrm{C}=\mathrm{C}$ stretching mode. The spectrogram is a time-frequency analysis using the sliding Fourier transform window and an artifact can appear in the spectrogram trace as a modulation of frequency and intensity that is caused by a beat 
between neighboring frequency modes. ${ }^{37}$ The modulation period corresponds to the inverse of the frequency difference between the neighboring modes. When the time width of the sliding window is much shorter than the modulation period of the artifact, the spectrogram trace is significantly contaminated by the artifact. The observed frequency modulation in Figure $4 \mathrm{~b}$ has a period of $500 \mathrm{fs}$, which corresponds to $67 \mathrm{~cm}^{-1}$. If the frequency modulation is caused by an artifact, neighboring frequency modes separated by $67 \mathrm{~cm}^{-1}$ should be observed in the Fourier power spectrum of the time-resolved difference absorption trace. However, the Fourier power spectrum calculated from the time-resolved trace in the region from 200 to $1200 \mathrm{fs}$ did not show a neighboring mode around $1500 \mathrm{~cm}^{-1}$ separated by $67 \mathrm{~cm}^{-1}$. It confirms that the frequency modulation observed in the spectrogram trace is not an artifact caused by the beat between neighboring modes, but reflects a real-time frequency change of the vibration frequency.

After photoisomerization of the retinal is completed, the frequency of the $\mathrm{C}=\mathrm{C}$ stretching mode was found to be modulated at a period of $\sim 500 \mathrm{fs}$. The frequency modulation reflects the wavepacket motion on the potential energy surface of the relevant electronic states of all of the ground state, the excited state, prime $\mathrm{Rh}$, and bathoRh, which appear after the photoexcitation. ${ }^{38,39}$ This value for octopus $\mathrm{Rh}$ is consistent with the period of $550 \mathrm{fs}$ observed in the wavepacket motion results on bovine $\mathrm{Rh}$ reported by Shank et al., ${ }^{40}$ and is considerably different from the period of $\sim 200 \mathrm{fs}$ in $\mathrm{bR}^{27}$ The frequency modulation reflects torsional motion around the $\mathrm{C}_{11}=\mathrm{C}_{12}$ double bond before thermalization in the all-trans structure. The change in the frequency can be ascribed to modulation of the bond length of the $\mathrm{C}_{11}=\mathrm{C}_{12}$ double bond using an empirical equation relating the bond length and vibration frequency. The empirical equation is obtained as follows.

The relationship between the bond-stretching force constant $k(\mathrm{dyn} / \mathrm{cm})$, bond length $d(\AA), \pi$ bond order $(P)$, and electronegativity $(\chi)$ was found by Gordy as follows: ${ }^{41}$

$$
k=a(P+1)\left(\frac{\chi}{d}\right)^{3 / 2}+b
$$

Here, $a$ and $b$ are empirical constants equal to $1.67 \times 10^{5}$ and $0.30 \times 10^{5}$, respectively, for stable molecules exhibiting their normal covalencies. Gordy has estimated the average deviation of $k$ calculated from $k$ values observed for 71 cases to be $1.84 \%$. The bond vibration frequency $\nu\left(\mathrm{cm}^{-1}\right)$ can then be related to the bond-stretching force constant $k(\mathrm{dyn} / \mathrm{cm})$ assuming an isolated oscillator as

$$
\nu=\left(\frac{1}{2 \pi^{2} c^{2} m}\right)^{1 / 2} k^{1 / 2}
$$

where $m$ and $c$ are the mass of the bonded atom and the velocity of light, respectively. ${ }^{42}$ Taking eqs 1 and 2, we can obtain the relation between the bond length $d$ and the vibration frequency $\nu$ as

$$
\nu=C\left[a(P+1)\left(\frac{x}{d}\right)^{3 / 2}+b\right]^{1 / 2}
$$

where $\chi=2.5$ and $C=1.66$. Dewar derived the relationship between the bond length $d$ and the bond order $P$ for single and double bonds as $d=1.489-0.151 \mathrm{P} .{ }^{43}$ For a single bond, $P$ is 0 , and for a double bond, $P$ is 1 . Using this equation, the observed frequency change in the $\mathrm{C}=\mathrm{C}$ stretching mode leads to a modulation in the bond length of the $\mathrm{C}_{11}=\mathrm{C}_{12}$ double bond of $\sim 11 \pm 2 \mathrm{~mA}$.

The assignment was certified by the direct observation of the real-time frequency of the $\mathrm{HOOP}$ mode and $\mathrm{C}=\mathrm{C}-\mathrm{H}$ in-plane bending mode as discussed below.

The in-plane $\mathrm{C}=\mathrm{C}-\mathrm{H}$ bending modes coupled with $\mathrm{C}-\mathrm{C}$ stretching modes appear at around $1200 \mathrm{~cm}^{-1}$. This is the socalled fingerprint region, which is very sensitive to chromophore conformation. In the observed spectrogram, the HOOP mode and $\mathrm{C}=\mathrm{C}-\mathrm{H}$ in-plane bending mode are merged until $\sim 200 \mathrm{fs}$, followed by the clear separation of the two modes. It elucidates that the rapid tortion along the HOOP coordinate finishes around $200 \mathrm{fs}$, which is the time when primeRh is reported to appear. ${ }^{7,32}$

The intensities of the HOOP mode and $\mathrm{C}=\mathrm{C}-\mathrm{H}$ in-plane bending mode are also modulated at a period of $\sim 500 \mathrm{fs}$, reflecting torsional motion around the $\mathrm{C}_{11}=\mathrm{C}_{12}$ double bond in thermalization from primeRh to bathoRh.

Therefore, when the system crosses CI at 80 fs after excitation, the 200-fs tortional motion along the HOOP coordinate is nearly half finished, and the system is still in the 11-cis configuration with partial deformation of the quasi planarity of the plane formed by $C_{11}=C_{12}-C_{13}$ bonds, i.e., the torsional motion around the $\mathrm{C}_{11}=\mathrm{C}_{12}$ double bond with 500-fs period is (partially) finished with the rotaion angle of about one-sixth $(=80 / 500)$ of $180^{\circ}$.

Figure $4 \mathrm{c}$ shows two-dimensional Fourier power spectra of the time-resolved difference absorption traces $\Delta A(t)$ over delay times ranging from 800 to 2000 fs. The signals centered at around $60 \mathrm{~cm}^{-1}$ confirm that these time-resolved traces are also modulated at a period of $\sim 500 \mathrm{fs}$, reflecting wavepacket motion at the same period.

In the case of bovine $\mathrm{Rh}$, the transition time from the $\mathrm{FC}$ state to CI is reported to be $50 \mathrm{fs}{ }^{44}$ which was estimated as $80 \mathrm{fs}$ for octopus Rh in the present work. For the other transitions with femtosecond and picosecond time constants in bovine $\mathrm{Rh}$, the results are similar to those observed in octopus $\mathrm{Rh}$ shown above. Comparing these results with our previous study on transient absorption of $\mathrm{bR}^{27}$ we can see that the transition from the FC state to $\mathrm{CI}$ proceeds faster in octopus $\mathrm{Rh}$ ( $80 \mathrm{fs})$ than in bR ( 200 fs).

\section{CONCLUSIONS}

In this work, we have measured time-resolved difference absorption spectra of octopus $\mathrm{Rh}$ using a sub-5-fs laser pulse with broadband multichannel detectors. The obtained data matrix has 128 data points in the 528-727-nm region with a 1.56-nm interval and 2100 data points between -100 and $2000 \mathrm{fs}$ with a 1-fs interval. The obtained time-resolved traces were fitted by a double-exponential function using a global fitting method over all probe wavelengths. The time constants of the processes following photoexcitation are comparable between the octopus $\mathrm{Rh}$ and bovine $\mathrm{Rh}$ results. However, in comparison with the model bR system, the transition time from the FC state to $\mathrm{CI}$ in octopus $\mathrm{Rh}$ were found to be $80 \mathrm{fs}$, being about 3 times shorter than in $\mathrm{bR}$, which we measured in transient absorption of $\mathrm{bR}^{27}$

The vibration dynamics of photoisomerization were studied by spectrogram analysis. At delay times shorter than $150 \mathrm{fs}$, the $\mathrm{C}=\mathrm{N}$ stretching mode appears and quickly disappears within 
$150 \mathrm{fs}$. This observation indicates that the primary event after photoexcitation of the octopus $\mathrm{Rh}$ is a deformation of the retinal configuration near the $\mathrm{C}=\mathrm{N}$ bond in the protonated Schiff base. An observed frequency modulation of the $\mathrm{C}=\mathrm{C}$ stretching mode at a period of $\sim 500$ fs was also observed, reflecting torsional motion around the $\mathrm{C}_{11}=\mathrm{C}_{12}$ double bond of primeRh before thermalization to bathoRh. This value is similar to the period of $550 \mathrm{fs}$ obtained for wavepacket motion observed in transient absorption measurement of bovine $\mathrm{Rh}^{40}$ By contrast, the modulation period for bR was observed to be $\sim 200 \mathrm{fs}$ in transient absorption measurement of $\mathrm{bR}^{27} \mathrm{On}$ the basis of the frequency change in the $\mathrm{C}=\mathrm{C}$ stretching mode, the bond length of the $\mathrm{C}_{11}=\mathrm{C}_{12}$ double bond was found to be modulated on the order of $\sim 10 \mathrm{~m} \AA$. The HOOP and $\mathrm{C}=\mathrm{C}-\mathrm{H}$ modes are merged until $200 \mathrm{fs}$, therefore the rapid torsion along the HOOP coordinate is thought to be half finished at the delay of $80 \mathrm{fs}$ when the system crosses CI. Their intensities were observed to be modulated at a period of $\sim 500 \mathrm{fs}$, again affected by the torsional motion around the $\mathrm{C}_{11}=\mathrm{C}_{12}$ double bond.

\section{AUTHOR INFORMATION}

\section{Corresponding Author}

*Address: Department of Electrophysics, National Chiao-Tung University, Hsinchu 300, Taiwan. Telephone: 88635712121 ext56197. Fax: 8863 5725230. E-mail: yabushita@mail.nctu. edu.tw.

\section{ACKNOWLEDGMENTS}

This work was supported by the Japan Science and Technology Agency (JST), National Science Council of the Republic of China, Taiwan (NSC 98-2112-M-009-001-MY3, NSC 99-2923M-009-004-MY3), and a grant from the Ministry of Education, Aiming for Top University (MOE ATU) Program at National Chiao-Tung University (NCTU). This work was also supported in part by a Grant-in-Aid for Scientific Research from the Japan Society for the Promotion of Science (JSPSGASR-14002003).

\section{REFERENCES}

(1) Yoshizawa, T.; Kito, Y. Nature 1958, 182, 1604-1605.

(2) Yoshizawa, T.; Wald, G. Nature 1963, 197, 1279-1286.

(3) Buchert, J.; Stefancic, V.; Doukas, A. G.; Alfano, R. R.; Callender, R. H.; Pande, J.; Akita, H.; Balogh-Nair, V.; Nakanishi, K. Biophys. J. 1983, 43, 279-283.

(4) Horiuchi, S.; Tokunaga, F.; Yoshizawa, T. Biochim. Biophys. Acta 1980, 591, 445-457.

(5) Eyring, G.; Curry, B.; Mathies, R.; Fransen, R.; Palings, I; Lugtenburg. J. Biochem. 1980, 19, 2410-2418.

(6) Kandori, H.; Shichida, Y.; Yoshizawa, T. Biochemistry (Moscow) 2001, 66, 1197-1209.

(7) Kukura, P.; McCamant, D. W.; Yoon, S.; Wandschneider, D. B.; Mathies, R. A. Science 2005, 310, 1006-1009.

(8) Ohtani, H.; Kobayashi, T.; Tsuda, M.; Ebrey, T. G. Biophys. J. 1988, 53, 17-24.

(9) Shichida, Y.; Matsuoka, S.; Yoshizawa, T. Photobiochem. Photobiophys. 1984, 7, 221-228.

(10) Sperling, W. In Biochemistry and Physiology of Visual Pigments; Langer, H, Ed.; Springer-Verlag: Heidelberg, 1973; pp 19-28.

(11) Fukada, Y.; Shichida, Y.; Yoshizawa, T.; Ito, M.; Kodama, A.; Tsukida, K. Biochemistry 1984, 23, 5826-5832.

(12) Mao, B.; Tsuda, M.; Ebrey, T. G.; Akita, H.; Balogh-Nair, V.; Nakanishi, K. Biophys. J. 1981, 35, 543-546.

(13) Becker, R. S.; Freedman, K. J. Am. Chem. Soc. 1985, 107, 14771485.
(14) Koyama, Y.; Kubo, K.; Komori, M.; Yasuda, H.; Mukai, Y. Photochem. Photobiol. 1991, 54, 433-443.

(15) Dartnall, H. J. A. Vision Res. 1967, 8, 339-358.

(16) Kobayashi, T.; Kim, M.; Taiji, M.; Iwasa, T.; Nakagawa, M.; Tsuda, M. J. Phys. Chem. B 1998, 102, 272-280.

(17) Zhu, L.; Kim, J.; Mathies, R. A. J. Raman Spectrosc. 1999, 30, $777-783$.

(18) Kandori, H.; Sasabe, H.; Nakanishi, K.; Yoshizawa, T.; Mizukami, T.; Shichida, Y. J. Am. Chem. Soc. 1996, 118, 1002-1005.

(19) Chosrowjan, H.; Mataga, N.; Shibata, Y.; Tachibanaki, S.; Kandori, H.; Shichida, Y.; Okada, T.; Kouyama, T. J. Am. Chem. Soc. 1998, 120, 9706-9707.

(20) Kandori, H.; Furutani, Y.; Nishimura, S.; Shichida, Y.; Chosrowjan, H.; Shibata, Y.; Mataga, N. Chem. Phys. Lett. 2001, 334, 271-276.

(21) Schoenlein, R. W.; Peteanu, L. A.; Mathies, R. A.; Shank, C. V. Science 1991, 254, 412-415.

(22) Kim, J. E.; Mathies, R. A. J. Phys. Chem. A 2002, 106, 85088515.

(23) Shim, S.; Dasgupta, J.; Mathies, R. A. J. Am. Chem. Soc. 2009, 131, 7592-7597.

(24) Yabushita, A.; Kobayashi, T. Biophys. J. 2009, 96, 1447-1461.

(25) Pollard, W. T.; Brito Crus, C. H.; Shank, C. V.; Mathies, R. A. J. Chem. Phys. 1989, 90, 199-208.

(26) Du, M.; Flemming, G. R. Biophys. Chem. 1993, 48, 101-111.

(27) Kobayashi, T.; Saito, T.; Ohtani, H. Nature 2001, 414, 531-534.

(28) Baltuska, A.; Kobayashi, T. Appl. Phys. B: Laser Opt. 2002, 75, 427-443.

(29) Kobayashi, T.; Shirakawa, A.; Matsuzawa, H.; Nakanishi, H. Chem. Phys. Lett. 2000, 321, 385-397.

(30) Shirakawa, A.; Sakane, I.; Takasaka, M.; Kobayashi, T. Appl. Phys. Lett. 1999, 74, 2268-2270.

(31) Hozwarth, A. R. Adv. Photosynth. Respir. 2004, 3, 75-92.

(32) Peteanu, L. A.; Schoenlein, R. W.; Qang, Q.; Mathies, R. A.; Shank, C. V. Proc. Natl. Acad. Sci. U.S.A. 1995, 9, 11762-11766.

(33) Meier, T.; Mukamel, S. Phys. Rev. Lett. 1996, 77, 3471-3474.

(34) Rutz, S.; Schreiber, E. Eur. Phys. J. D 1998, 4, 151-158.

(35) Smith, S. O.; Braiman, M. S.; Myers, A. B.; Pardoen, J. A.; Courtin, J. M. L.; Winkel, C.; Lugtenburg, J.; Mathies, R. A. J. Am. Chem. Soc. 1987, 109, 3108-3125.

(36) Rothschild, K. J.; Marrero, H. Proc. Natl. Acad. Sci. U.S.A. 1982, 79, 4045-4049.

(37) Kahan, A.; Nahmias, O.; Friedman, N.; Sheves, M.; Ruhman, S. J. Am. Chem. Soc. 2007, 129, 537-546.

(38) Pollard, W. T.; Lee, S.-Y.; Mathies, R. A. J. Chem. Phys. 1990, 92, 4012-4029.

(39) Jean, J. M.; Fleming, G. R. J. Chem. Phys. 1995, 103, 2092-2101.

(40) Wang, Q.; Schoenlein, R. W.; Peteanu, L. A.; Mathies, R. A.; Shank, C. V. Science 1994, 266, 422-424.

(41) Gordy, W. J. Chem. Phys. 1946, 14, 305-320.

(42) Baughman, R. H.; Witt, J. D.; Yee, K. C. J. Chem. Phys. 1974, 60, 4755-4759.

(43) Dewar, M. J. S. Hyperconjugation; Modern Concepts in Chemistry; Ronald Press: New York, 1962; Chapters 3 and 4, pp 48-70.

(44) Kochendoerfer, G. G.; Mathies, R. A. J. Phys. Chem. 1996, 100, 14526-14532. 